\title{
ASCO 2009: What's New in Breast Cancer Therapy?
}

\author{
Chair: Laura Biganzolia \\ Participants: Hervé Bonnefoi ${ }^{b}$ Fatima Cardoso $^{c}$ Robert Coleman ${ }^{d}$ Beat Thürlimann ${ }^{\mathrm{e}}$ \\ a 'Sandro Pitigliani' Medical Oncology Unit, Department of Oncology, Hospital of Prato, Tuscany Cancer Institute, Prato, Italy \\ ${ }^{\mathrm{b}}$ Institut Bergonie, Bordeaux, France \\ ${ }^{c}$ Medical Oncology \& Translational Research, Jules Bordet Institute, Brussels, Belgium \\ ${ }^{d}$ Academic Unit of Clinical Oncology, Weston Park Hospital, Sheffield, UK

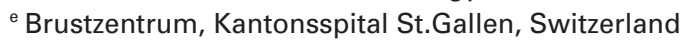

\section{Question 1: PARP Inhibitors in Triple Negative Breast Cancer. Still a Promise or a Reality?}

Bonnefoi: Definitely PARP (poly(ADR-ribose)polymerase) inhibitors are a reality both in combination with chemotherapy and as a single agent - see the phase 1 trial published in the New England Journal of Medicine [1] (we don't often see a phase 1 in the NEJM). In combination with chemotherapy in patients with metastatic breast cancer a phase 3 trial is now ongoing in the US. Other trials are under development in Europe. Many questions need to be answered: Which is the best chemotherapy to combine with a PARP inhibitor? How to define BRCAness in clinic? How to go beyond breast and ovarian cancer indications?

Cardoso: They are still only a promise but with very high hopes regarding their potential to help treat this aggressive form of breast cancer. A phase 3 trial should be launched without delay to provide the level of evidence needed to have this treatment approved and reimbursed, if the very promising phase 2 data are confirmed.

Coleman: Defective DNA repair has been elegantly demonstrated to be a major abnormality in hereditary and basal-like triple negative tumours [2,3]. PARP inhibitors exploit this defect and, either alone or in combination with platinum based chemotherapy, have been shown in two studies presented at ASCO 2009 to have remarkable activity $[4,5]$. Indeed in the randomised phase 2 study presented by O'Shaughnessy [2], significant benefit not only in response rates and PFS were seen, but also overall survival was significantly improved with BSI-201 in combination with carboplatin and gemcitabine compared with the same chemotherapy agents alone, despite cross-over on progression in approximately $40 \%$. The results of this trial are so striking that they almost challenge the dogma for a phase 3 study of chemotherapy + PARP inhibitors versus chemotherapy alone to demonstrate efficacy and achieve regulatory approval.

Thürlimann: I think they are a promise as of today, but a realistic promise. We urgently need adequate trials to establish the clinical position for these drugs which showed encouraging activity. Translational research in association with these clinical investigations especially in the adjuvant setting are of paramount importance.

\section{Question 2: Do We Have More Insight in the Use of Bevacizumab in Metastatic Breast Cancer?}

Bonnefoi: The results of RIBBON-1 [6] confirm the benefit seen in previous trials. A new combination can be added on the list in first-line therapy: bevacizumab + capecitabine. However, the theme of the ASCO meeting was 'Personalizing Cancer Care': chemotherapy combined with bevacizumab is one of the options for patients with metastatic breast cancer, but not the only one.

Cardoso: The promise of anti-angiogenic therapy has taken too long to materialise. Bevacizumab seems to be only mildly active in metastatic breast cancer and only when given early on, specifically as first-line treatment. It is also associated with potentially serious and even fatal side effects, and with too high costs. It is therefore indispensible to keep the research efforts focused on finding predictive markers of response/resistance that may help us to better select the patients who can really benefit from this agent.

\section{KARGER}

Fax +497614520714

Information@Karger.de

www.karger.com (c) 2009 S. Karger GmbH, Freiburg
Laura Biganzoli, MD

'Sandro Pitigliani' Medical Oncology Unit

Department of Oncology, Hospital of Prato

Tuscany Cancer Institute

Piazza dell'Ospedale 2, 59100 Prato, Italy

lbiganzoli@usl4.toscana.it 
Coleman: Presentation of the RIBBON-1 data at ASCO 2009 demonstrated that bevacizumab clearly augments the efficacy of a range of single-agent chemotherapy regimens including anthracyclines, paclitaxel and capecitabine. The results add to the previously positive E2100 and AVADO trials. However, the challenge remains that we still have no validated biomarker to identify patients most likely to benefit, and the benefits are quite modest when compared with other approved targeted agents such as trastuzumab. Until bevacizumab becomes a truly targeted treatment, rather than an option for all, it will remain unaffordable for the majority of health care systems and not a cost effective advance in the management of advanced malignancy. As a proof of principle and demonstration of broad activity with acceptable toxicity, RIBBON-1 is an important study; we now need to use the biological materials collected in the trials to define the responsive sub-population.

Thürlimann: We have some insight as first-line combination therapy but the role of bevacizumab has not sufficiently been explored. This is especially the case for the optimal duration of the first-line treatment.

\section{Question 3: Bisphosphonates as Anticancer Treatment in Early Breast Cancer. Are We Ready for Clinical Practice?}

\section{Bonnefoi: See Rob Coleman.}

Cardoso: Not yet. A single, relatively small adjuvant trial should not lead to a change in clinical practice, except in special circumstances, which in my opinion it is not the case. A confirmatory study is necessary. However, if confirmed these are very exciting data since they represent a paradigm change and the 'discovery' of a new class of anticancer agents. The biological rationale exists and, since these agents will also be of great value for bone health, particularly in postmenopausal women receiving adjuvant aromatase inhibitors, their place in the adjuvant treatment of breast cancer will certainly strongly increase. However several important questions remain unanswered, particularly regarding the frequency of administration, dosing and type of bisphosphonate. These questions have also important economic implications that should not be overlooked.

Coleman: Metastatic bone disease results from the interactions between cancer cells in the bone marrow microenvironment and normal bone cells, thereby providing the rationale for bone-targeted therapies. Bisphosphonates interrupt the vicious cycle within the marrow microenvironment and may also have direct effects on tumour cells, especially when administered in combination with chemotherapy [7]. Recent data with zoledronic acid, notably from the ABCSG-12 trial
[8], supported by previous studies with oral clodronate $[9,10]$ suggest that bone targeted treatments may indeed modify the course of the disease and reduce recurrence rates. However, ABCSG-12 studied a very specific population of oestrogen receptor positive $(\mathrm{ER}+)$ premenopausal women receiving endocrine treatment and the results may not apply to the broader range of breast cancer patients encountered in clinical practice. The results of ongoing large metastasis prevention trials in breast cancer (NSABP-B34, AZURE) are required before routine use of adjuvant bisphosphonates, outside the context of clinically relevant treatment induced bone loss, can be recommended.

Thürlimann: We are not yet completely ready for clinical practice. We need convincing and unequivocal evidence for their use in different patient and treatment settings. ABCSG12 is a paradigmatic trial in a small, well selected population with specific and partially experimental treatments. It is a positive 'proof of principle'. The following larger trials will soon give more information in broader populations with 'standard' treatments and with a higher event rate.

\section{Question 4: Gennomic Signature and Early Breast Cancer. When and Why?}

Bonnefoi: See Fatima Cardoso.

Cardoso: The accurate identification of the patients that need adjuvant chemotherapy is one of the most important dilemmas in clinical practice. The commonly used clinico-pathological factors are important but not sufficient to help us in this selection. New tools are therefore of crucial importance. Two tests are in the most advanced phase of development: MammaPrint and Oncotype DX. Both are undergoing prospective validation in large adjuvant trials: MINDACT and TAILORx. Until these tools have achieved level 1 evidence from prospective validation, they should not be widely used in clinical practice, in the same way as a new drug is very rarely approved on the basis of phase 2 data only. I therefore disagree with the recent endorsement of these tests by the St. Gallen consensus, even if it was done only for cases where doubt persists. This endorsement should have waited for the end of recruitment of the important prospective trials. All these new prognostic genomic tools seem to reflect the same biological phenomenon, which is proliferation. This is probably also the reason why they are also associated with a better response to chemotherapy. New signatures reflecting other important pathways need to be developed and combined with the existing ones to increase their prognostic potential.

Coleman: We are witnessing an explosion of information on molecular phenotyping and generation of genetic signatures in breast cancer aimed at either accurately defining prognosis 
or predicting response to treatment. Clearly there is a great need to characterise individual tumours accurately to enable treatment to be personalised, thereby minimising the risk of overtreatment and utilising the best combination of agents in an individual patient. Currently available tools (Oncotype DX and MammaPrint) appear to accurately define a low-risk population who do not require chemotherapy. However, further prospective validation of both techniques is needed and in progress (TAILORx and MINDACT), while at present, predictive signatures remain a research tool and are hypothesis generating only. It will also be important to show that these complex and expensive profiling methods truly do out-perform routine pathological assessment of tumour grade with high-quality immunohistochemistry for ER, PgR, HER2 and proliferation (Ki-67 or similar).

Thürlimann: We do not routinely use genomic signatures. We are in the lucky position to have access to high-quality pathology including continuous external quality assurance. We acknowledge that in other environments they may play a more important role for the daily routine. We should await the results of ongoing prospective randomised trials before we draw new conclusions.

\section{Question 5: Aromatase Inhibitors in the Adjuvant Setting. Upfront or Not Upfront?}

\section{Bonnefoi: See Beat Thürlimann.}

Cardoso: The recent results of the BIG 1-98 trial provide the rationale for starting more often with an aromatase inhibitor (AI) upfront since they have shown that one can safely switch to tamoxifen after two years of an AI, in case tolerance is not optimal. Tolerability of AIs is a huge problem in clinical practice, particularly due to arthralgia and myalgia. The possibility of prescribing the 'reverse' sequence allows women who do not optimally tolerate the AI to safely shift to tamoxifen, while at the same time receiving the AI during the first two years when the rate of relapse is higher.

Coleman: Trials of the aromatase inhibitors in postmenopausal ER+ breast cancer have consistently shown improved efficacy over tamoxifen and a favourable toxicity profile with the exception of more frequent musculoskeletal side effects and accelerated bone loss. However, debate continues about whether an AI should replace or be added in sequence to treatment with tamoxifen. This is based on both the biologi- cal question as to whether two endocrine approaches are better than one as well as health economics. Both up-front and sequence/switching strategies have been shown to clearly improve disease-free survival (DFS) and also produce small survival gains that are of borderline statistical significance. The BIG1-98 trial confirms that an AI should be part of routine treatment for the vast majority of women with postmenopausal ER+ breast cancer. However, this trial has failed to show any significant difference in outcome between the immediate or sequencing strategies. Only the TEAM trial comparing 5 years exemestane to a tamoxifen-exemestane sequence in $>9000$ women is powered to definitively address this question. It is anticipated that the 5 year results from TEAM will be available in 2010. Until then a strategy of immediate AI for intermediate- to high-risk patients and a sequence of tamoxifen - AI or AI - tamoxifen for low- to intermediate-risk patients remains a reasonable approach.

Thürlimann: After the recent presentations in San Antonio and St. Gallen we clearly prefer the upfront use of AI if they are prescribed.

\section{Participants}

Hervé Bonnefoi, MD

Institut Bergonie

229, cours de l'Argonne

33076 Bordeaux CEDEX, France

Bonnefoi@bergonie.org

Fatima Cardoso, MD

Medical Oncology \& Translational Research

Jules Bordet Institute

Boulevard de Waterloo, 125

1000 Brussels, Belgium

Tel. +3225413082

Fax +32 25380858

fatima.cardoso@bordet.be

Robert Coleman, MD

Academic Unit of Clinical Oncology

Weston Park Hospital

Sheffield, UK

r.e.coleman@sheffield.ac.uk

Prof. Dr. med. Beat Thürlimann

Kantonsspital St. Gallen

Brustzentrum

9007 St. Gallen, Switzerland

Tel. +41 714941177

Fax +41 714946368

beat.thuerlimann@kssg.ch 


\section{References}

1 Fong PC, Boss DS, Yap TA, Tutt A, Wu P, et al.: Inhibition of poly(ADP-ribose) polymerase in tumors from BRCA mutation carriers. N Engl J Med 2009; 361:123-134.

2 Farmer H, McCabe N, Lord CJ, Tutt AN, et al.: Targeting the DNA repair defect in BRCA mutant cells as a therapeutic strategy. Nature 2005;43:917-921.

3 Bryant HE, Schultz N, Thomas HD, Parker KM, Flower D, et al.: Specific killing of BRCA2-deficient tumours with inhibitors of poly(ADP-ribose) polymerase. Nature 2005;434:913-917. Erratum in: Nature 2007;447:346.

4 O'Shaughnessy J, Osborne C, Pippen J, Yoffe M, Patt D, et al.: Efficacy of BSI-201, a poly (ADPribose) polymerase-1 (PARP1) inhibitor, in combination with gemcitabine/carboplatin $(\mathrm{G} / \mathrm{C})$ in patients with metastatic triple-negative breast cancer (TNBC): Results of a randomized phase II trial. J Clin Oncol 2009;27:18s (suppl) abstr 3.
5 Tutt A, Robson M, Garber JE, Domchek S, Audeh MW, et al.: Phase II trial of the oral PARP inhibitor olaparib in BRCA-deficient advanced breast cancer. J Clin Oncol 2009;27:18s (suppl) abstr CRA501.

6 Robert NJ, Dieras V, Glaspy J, Brufsky A, et al.: RIBBON-1: Randomized, double-blind, placebocontrolled, phase III trial of chemotherapy with or without bevacizumab (B) for first-line treatment of HER2-negative locally recurrent or metastatic breast cancer (MBC). J Clin Oncol 2009;27:15s (suppl) abstr 1005.

7 Ottewell PD, Mönkkönen H, Jones M, Lefley DV, et al.: Antitumor effects of doxorubicin followed by zoledronic acid in a mouse model of breast cancer. J Natl Cancer Inst 2008;100:1167-1178.
8 Gnant M, Mlineritsch B, Schippinger W, LuschinEbengreuth G, Pöstlberger S, et al.: Endocrine therapy plus zoledronic acid in premenopausal breast cancer. N Engl J Med 2009;360:679-691. Erratum in: N Engl J Med 2009;360:2379.

-9 Powles T, McCroskey E, Paterson A: Oral bisphosphonates as adjuvant therapy for operable breast cancer. Clin Cancer Res 2006;12:6301s-6304s.

10 Diel IJ, Jaschke A, Solomayer EF, Gollan C, Bastert $\mathrm{G}$, et al.: Adjuvant oral clodronate improves the overall survival of primary breast cancer patients with micrometastases to the bone marrow: a longterm follow-up. Ann Oncol 2008;19:2007-2011. 